\title{
Rheology of a long lava flow at Pavonis Mons, Mars
}

\author{
S. M. Baloga \\ Proxemy Research, Laytonsville, Maryland, USA
}

P. J. Mouginis-Mark

Hawaii Institute of Geophysics and Planetology, University of Hawaii, Honolulu, Hawaii, USA

L. S. Glaze

Proxemy Research, Laytonsville, Maryland, USA

Received 16 September 2002; revised 4 March 2003; accepted 2 April 2003; published 3 July 2003.

[1] Dimensions of lava flows can be used to unravel the relative roles of viscosity changes and concurrent formation of levees, stationary margins, and stagnant zones. This approach is applied to data derived from the Mars Orbiter Laser Altimeter (MOLA) experiment for a long lava flow on the plains north of Pavonis Mons, Mars. We obtain a formula for the relative change in viscosity on the basis of a steady state Newtonian flow rate. Our approach features a new length scale that describes the transfer of lava from the active advancing component to passive components. This length scale can be determined from planetary image and topographic data by estimating the volume fraction of lava contained in flow margins relative to the total flow volume. We find only modest changes in viscosity over the distal $175 \mathrm{~km}$ of the Pavonis flow. Allowing the flow to also lose volume through degassing (resulting in a density increase) does little to affect the overall viscosity change. Thickening and widening of the flow with distance are as expected for a single coherent, isothermal, viscous flow. This dynamic regime features a balance between the formation of an outer skin and shedding of lava into stationary zones. Requirements for attaining such a regime include a thick flow, shallow slopes over extended distances, and preexisting surface roughness that is small compared to flow thickness. This style of emplacement may explain why many of the long, thick sheet-like flows on the plains of Mars often exhibit an unexpected lack of thickening with distance. INDEX TERMS: 5480 Planetology: Solid Surface Planets: Volcanism (8450); 5464 Planetology: Solid Surface Planets: Remote sensing; 8450 Volcanology: Planetary volcanism (5480); 8429 Volcanology: Lava rheology and morphology; KEYWORDS: Pavonis, lava flow, channel, Mars, viscosity, rheology

Citation: Baloga, S. M., P. J. Mouginis-Mark, and L. S. Glaze, Rheology of a long lava flow at Pavonis Mons, Mars, J. Geophys. Res., 108(E7), 5066, doi:10.1029/2002JE001981, 2003.

\section{Introduction}

[2] During the emplacement of a lava flow, three physical processes can be important: 1) changes in viscosity or other specifications of internal resistance to flow, 2) the formation of channels, levees, or stationary margins that divide the flow into active and inactive components, and 3) a loss of volatiles that causes a change in the density of the lava [e.g., Walker, 1973; Malin, 1980; Sparks and Pinkerton, 1978; Moore, 1987; Crisp and Baloga, 1994; Crisp et al., 1994; Keszthelyi and Self, 1998; Zimbelman, 1998]. The loss of lava volume from an active flow, with concurrent changes in flow resistance, was treated recently by Baloga et al. [1998], and applications to planetary flows appear in the work of Glaze and Baloga [1998]. Density changes with different types of flow rates and rheologic changes are treated by Baloga et al. [2001].

Copyright 2003 by the American Geophysical Union. 0148-0227/03/2002JE001981\$09.00
[3] It is particularly difficult to determine the relative roles of these processes because they can occur simultaneously in active flows. Here we use a simple model of lava flow emplacement and show how the dimensions of lava flows can be used to unravel the relative roles of viscosity changes and the concurrent formation of levees, stationary margins and stagnant zone. A similar approach can be applied to density changes while the flow is active.

[4] We apply this approach to dimensional and slope data derived from the Mars Orbiter Laser Altimeter (MOLA) experiment for a long, easy to identify lava flow on the plains north of the Pavonis Mons volcano on Mars $\left(10^{\circ} \mathrm{N}, 245^{\circ} \mathrm{E}\right)$. This flow is a well-defined solitary lobe that originated close to the crest of the ridge joining Pavonis and Ascraeus Montes, and can be distinguished for almost $175 \mathrm{~km}$ with significant evidence of channelization (Figure 1).

[5] Our application here assumes a Newtonian flow rate, although the approach could be used with any other flow rate [Baloga et al., 2001, and references therein]. Thus any 


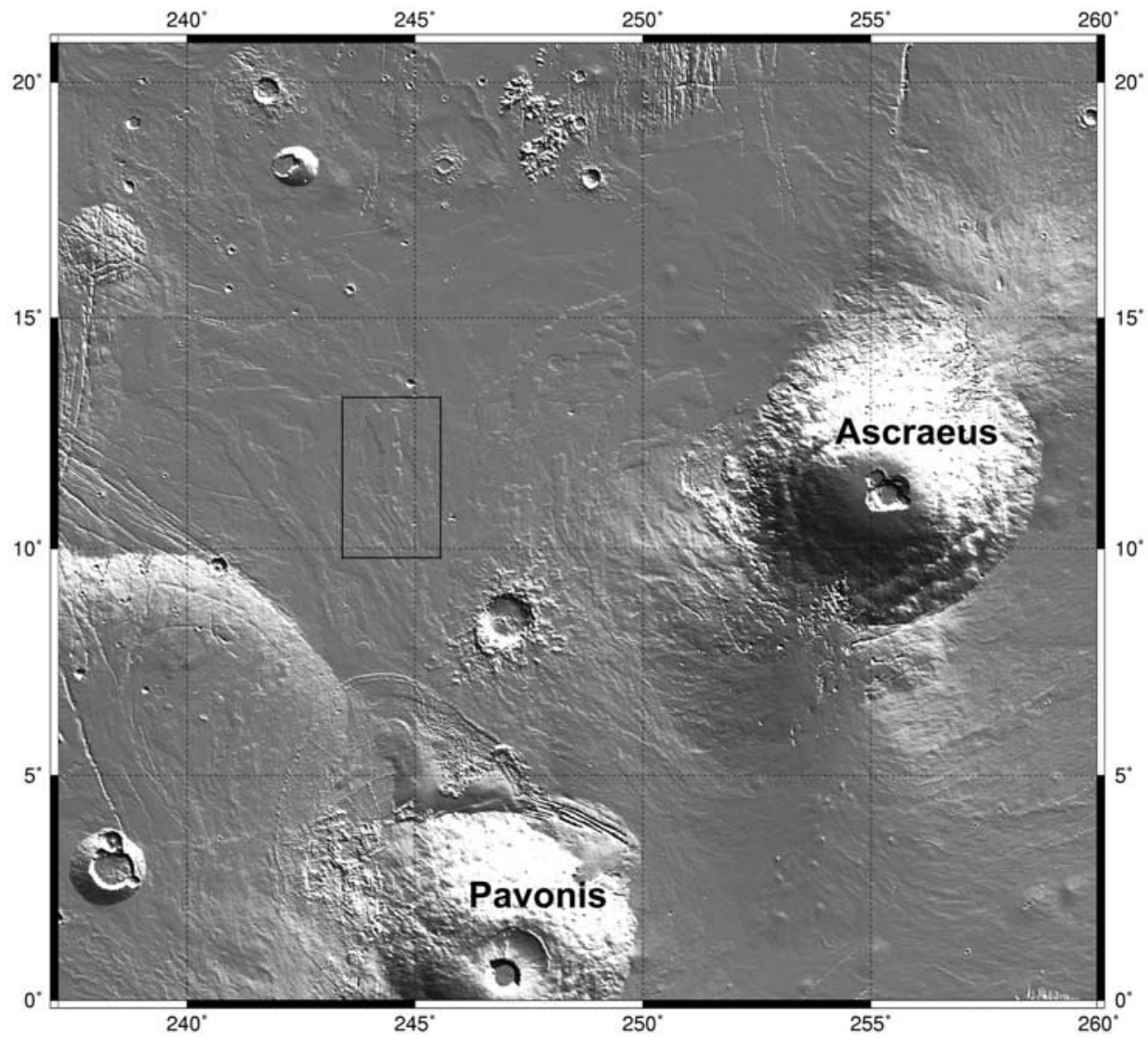

Figure 1. Shaded relief image of part of the Tharsis region, showing the location of the lava flow investigated here in relation to Pavonis and Ascraeus Montes. The $\sim 100 \times 200 \mathrm{~km}$ box indicates the portion of the lava flow investigated and corresponds to the area shown in Figure 3. Base image derived by Mike Carr of the U.S. Geological Survey.

systematic change in the thickness of a flow, beyond that anticipated from changes in the slope and width, is attributed to a change in the bulk viscosity of the lava. As a reference, we first look at the special case when there is no channelization and no density change. We then go to the more complex case when stationary margins are formed while the medial part of the flow remains active. Here we do not explicitly address the time dependent details of a concurrent density change while the flow is active. However, in the discussion, we develop some elementary steady state inferences and identify needs for subsequent theoretical and field research. In the conclusion, we summarize the results of our applications and suggest the inferred scenario of emplacement for the Pavonis flow studied here.

\section{Governing Equations}

[6] Our goal here is to develop a formalism for estimating viscosity changes when there is a loss of volume from the active part of the flow. Either the loss to stationary flow components or density changes along the flow path can mask an increase in viscosity. The influence of margin building and degassing during emplacement is to flatten the thickness profile. Failure to account for these processes could lead to significant underestimates of the actual change in viscosity.

[7] Processes influencing the final dimensions of a lava flow are shown schematically in Figure 2 [Moore, 1982,
1987; Lipman and Banks, 1987; Pinkerton and Wilson, 1994]. As the flow advances there may be a loss of lava mass and volume from the active medial part to inactive parts that may take the form of stationary margins, levees,

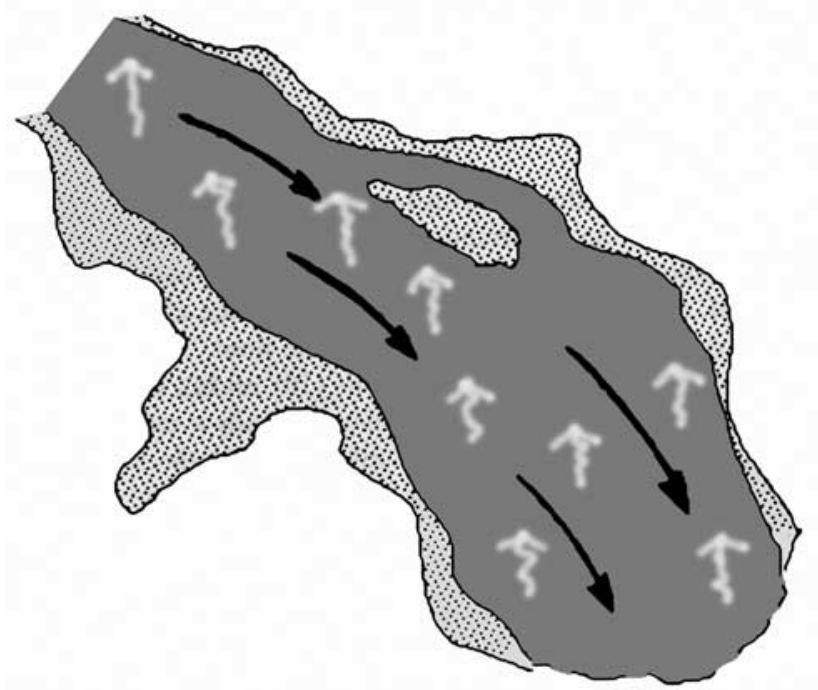

Figure 2. Schematic showing active and passive components, loss of volatiles, and change of width. 
Table 1. Notation

\begin{tabular}{ll}
\hline Variable & \multicolumn{1}{c}{ Definition } \\
\hline$h$ & Flow thickness $(\mathrm{m})$ \\
$L$ & Final flow length $(\mathrm{m})$ \\
$L_{g}$ & Length scale for volatile loss $(\mathrm{m})$ \\
$L_{m}$ & Length scale for mass loss $(\mathrm{m})$ \\
$Q$ & Volume flow rate $\left(\mathrm{m}^{3} \mathrm{~s}^{-1}\right)$ \\
$Q_{d}$ & Volume flow rate, degassing case $\left(\mathrm{m}^{3} \mathrm{~s}^{-1}\right)$ \\
$t$ & Time (s) \\
$t_{a}$ & Time for flow front to reach $x(\mathrm{~s})$ \\
$u$ & Flow velocity $\left(\mathrm{m} \mathrm{s}^{-1}\right)$ \\
$V_{d}$ & Total volume discharged $\left(\mathrm{m}^{3}\right)$ \\
$V_{m}$ & Volume in stationary margins $\left(\mathrm{m}^{3}\right)$ \\
$w$ & Flow width (m) \\
$x$ & Distance (m) \\
$\Gamma$ & Time to reach final length $(\mathrm{s})$ \\
$\theta$ & Underlying slope $\left({ }^{\circ}\right)$ \\
$\lambda_{g}$ & Volatile loss rate constant $\left(\mathrm{s}^{-1}\right)$ \\
$\lambda_{m}$ & Mass loss rate constant $\left(\mathrm{s}^{-1}\right)$ \\
$\nu$ & Kinematic viscosity $\left(\mathrm{m}^{2} \mathrm{~s}^{-1}\right)$ \\
$\rho$ & Density of lava $\left(\mathrm{kg} \mathrm{m}^{-3}\right)$ \\
$\Phi_{g}$ & Volatile loss function \\
$\Phi_{m}$ & Mass loss function \\
\hline &
\end{tabular}

overbank deposits, and stagnant zones. As is well-known, the viscosity often increases significantly along the flow path. If the lava degasses, its density will increase, effectively counteracting the thickening tendency of a viscosity increase [Baloga et al., 2001]. Finally, most unconfined flows feature variations in the total flow width, the width of the active medial component, and the underlying slope.

\subsection{Active Component of the Flow}

[8] We first focus on the conservation of flow volume for the active part of the flow. See Table 1 for definitions of mathematical symbols. Both the mass loss to the passive components of the flow and the density change due to volatile losses are assumed to be rate processes. We formally express both of these processes as loss functions in proportions $\boldsymbol{\Phi}_{m}$ and $\boldsymbol{\Phi}_{g}$ to the control volume. With this approach, $\boldsymbol{\Phi}_{m}$ and $\boldsymbol{\Phi}_{g}$ are dimensionless functions and the rate constants $\lambda_{m}$ and $\lambda_{g}$ have the conventional dimensions of time ${ }^{-1}$.

[9] The functions $\boldsymbol{\Phi}_{m}$ and $\boldsymbol{\Phi}_{g}$ describe how mass and volatiles are lost from the active part of the flow. At present little is known about these functions and below we will make some simplifying assumptions. The formulation of the equations here follows prior work in the literature [Baloga et al., 1998, 2001; Glaze and Baloga, 1998]. Here we only note the special considerations that apply to this more general formulation.

[10] Local conservation for a control volume $d V=h w d x$ in the active component of the flow is expressed by

$$
w \frac{\partial h}{\partial t}+\frac{\partial Q}{\partial x}=-\left(\lambda_{m} \Phi_{m}+\lambda_{g} \Phi_{g}\right) w h
$$

where $Q=Q(x, t)=u(x, t) h(x, t) w(x)$ is an arbitrary volumetric flow rate. The right hand side of (1) indicates that volume is lost from the active component as a rate process, as can be seen from the analysis of its dimensions. In (1), losses due to both margin formation and degassing are considered important. However, to simultaneously determine the change in density due to degassing and the flow thickness profile, we need to add a mass conservation equation. The solution of this more complicated system is beyond the scope of this paper. The implications of volume loss due to degassing are presented in the discussion. Thus the remainder of this section considers only the volume loss due to the removal of material from the active component to stationary margins.

[11] There is some information presently available on the rate constants in (1) from terrestrial and planetary applications [e.g., Baloga et al., 1998, 2001; Glaze and Baloga, 1998]. However, it is more convenient to deal with length scales representing these processes. Such length scales can readily be compared with the actual lengths of flows. Thus we make the definition

$$
L_{m}=\frac{Q_{o}}{\lambda_{m} w_{o} h_{o}}=\frac{u_{o}}{\lambda_{m}}
$$

where the " $O$ " subscripts indicate some starting location along the path of the flow. The length scale in (2) is the primary measure we use here to characterize the importance of mass losses to stationary margins as the flow advances. When $\lambda_{m}=0$, there is no construction of stationary margins. This is indicated by $L_{m}=\infty$.

[12] To obtain governing equations that can be solved explicitly from (1), we first evoke the assumption of steady state, i.e., a constant volumetric flow rate at the starting location along the flow path. Time-dependent effects are potentially very significant. But such considerations require additional assumptions and increase the complexity of the mathematics significantly [Baloga and Pieri, 1986; Baloga, 1987]. The influence of time dependence will be reserved for future analyses.

[13] We must also describe how mass losses occur by prescribing some specific form for $\boldsymbol{\Phi}_{m}$. Since little is presently known about this process, we make the simplest assumptions possible. We assume that the rate of volume loss at any point along the flow path is a constant. With this simplifying assumption, (1) reduces to

$$
\frac{d Q}{d x}=-\lambda_{m} w_{o} h_{o}
$$

This equation has the elementary solution

$$
Q(x)=Q_{o}\left(1-\frac{x}{L_{m}}\right)
$$

From (4), we will show how to develop inferences about rheologic change from flow thickness, width, and underlying slope data when $Q$ is given explicitly in terms of these variables. Typical flow rate forms expressing $Q$ in terms of $h, w$, and the slope are associated with a Newtonian, Bingham, or basal-glide dependencies on the thickness of the flow [e.g., Baloga et al., 2001, and references therein].

[14] Here, we use the Newtonian flow rate dependence,

$$
Q(x)=\frac{h^{3}(x) w(x) \sin \theta(x)}{3 \nu(x)}
$$


We then solve (4) with (5) for the relative viscosity,

$$
\frac{\nu(x)}{\nu_{o}}=\frac{\sin \theta(x) h(x) w(x)}{\sin \theta_{o} h_{o}^{3} w_{o}\left(1-x / L_{m}\right)} .
$$

Such a formula is often used to estimate relative viscosity changes for planetary flows [e.g., Glaze and Baloga, 1998]. However, the unknown parameter $L_{m}$ must be evaluated before we can use the dimensional data to estimate the viscosity change.

[15] In summary, the primary assumptions implicit in the use of (6) are as follows. First, systematic changes in the thickness and width of the flow are interpreted here as responses of the flow to changes in the preexisting topography or the bulk viscosity of the lava. Second, the rate of volume loss to the stationary margins is taken as a constant at all locations along the flow path. Thus the contribution to stationary components is proportional to the length of time they are supplied with lava. Finally, all time-dependent dynamical considerations are ignored. Each of these assumptions are stringent and further investigations are required.

\subsection{Passive Component of the Flow}

[16] We now turn our attention to the losses from the active component of the flow that construct stationary margins and other stagnant features. All of the volume discharged from the vent must be accounted for in either the active or stationary components when there is no degassing. This links the relative volumes of the active and passive components to the total volume discharged. In effect, this link provides a means for evaluating the unknown length scale $L_{m}$ appearing in (6).

[17] We now derive a method for obtaining the length scale $L_{m}$ when an estimate of the apportioning of the active and passive components is available.

[18] The time for the flow front to arrive at a distance $x$ is

$$
t_{a}(x)=\int_{o}^{x} \frac{d x}{u(x)}=\int_{o}^{x} \frac{h(x) w(x) d x}{Q(x)}=\frac{1}{Q_{o}} \int_{o}^{x} \frac{h(x) w(x) d x}{1-x / L_{m}} .
$$

When $L_{m}=\infty$, (7) reduces to a simple statement of volume conservation:

$$
Q_{o} t_{a}(x)=\int_{o}^{x} h w d x
$$

From (7) we can see the influence of the losses from the active component on the time of transit to any fixed distance. As $L_{m}$ becomes less and less, the more vigorous these processes are and the time of transit increases. Physically interpreted, lava is removed from the active component due to margin building at higher rates at each location along the path of the flow as $L_{m}$ decreases. This diminishes the flow rate at each step, thus engendering longer and longer transit times as $L_{m}$ decreases.

[19] Another way to look at the denominator in (7) is that it accounts for the fact that not all the volume discharged resides in the active component of the flow when margins are being constructed. The denominator, in effect, corrects for this so that all volume is accounted for between the two components at all times.

[20] If we consider a strip of thickness $d x$ at some distance downstream, $x$, then the total volume loss from that strip is given by

$$
d V_{m}=\lambda_{m} w_{o} h_{o} d x\left(\Gamma-t_{a}(x)\right)
$$

where we define $\Gamma$ as the time required for the flow front to reach its maximum extent, $L$. The difference in times on the right hand side of (9) thus starts the loss to the stationary component at the location of $d x$ only when the flow front arrives at $t_{a}(x)$.

[21] By integrating (9) over the length of the flow, we find the total volume of material in the stationary margins:

$$
V_{m}=\lambda_{m} h_{o} w_{o} \Gamma \int_{0}^{L}\left(1-\frac{t_{a}(x)}{\Gamma}\right) d x .
$$

The time for the flow front to attain its maximum extent can be written as

$$
\Gamma=\frac{V_{d}}{Q_{o}}=\frac{1}{Q_{o}} \int_{0}^{L} \frac{h(x) w(x)}{1-x / L_{m}} d x
$$

where $V_{d}$ is the total volume discharged, no matter where it goes by the time the flow front reaches $L$.

[22] For our present purposes, we are interested simply in the total allocation of lava into the active and passive components. These are quantities that can be estimated from the MOLA and imaging data available at present. Ergo, we can write the ratio of volume in the stationary margins to the total volume of lava erupted as

$$
\frac{V_{m}}{V_{d}}=\frac{L}{L_{m}}\left[1-\frac{1}{L} \int_{0}^{L} \frac{t_{a}(x)}{\Gamma} d x\right]
$$

where

$$
\frac{t_{a}(x)}{\Gamma}=\frac{\int_{0}^{x} \frac{h(x) w(x)}{1-x / L_{m}} d x}{\int_{0}^{L} \frac{h(x) w(x)}{1-x / L_{m}} d x} .
$$

Equations (12) and (13) provide the key to obtaining the unknown parameter $L_{m}$ when estimates of the relative volumes of the active and passive components are known. By inspection of these two equations, it is evident that there is only dimensional data and $L_{m}$ present.

[23] The implicit nature of $L_{m}$ in (12) and (13) makes it impossible to isolate it analytically. However, given the dimensional data for any flow, the full range of $L_{m}$ can be inserted into these equations, producing a single curve for the relative volume in the stationary components. Then from the measured or estimated fraction of volume components, 
$L_{m}$ can be taken from the flow-specific curve. Once this parameter is obtained, viscosity estimates can be computed from (6). This procedure is illustrated below for the flow shown in Figure 3.

\section{Analysis of the Pavonis Flow}

[24] The lava flow investigated here originates from the rift zone that joins the volcanoes Pavonis and Ascraeus Montes (Figure 1). It is one of at least a dozen that can be seen to flow north from the rift zone. These north trending flows are all mapped as unit Apm1 by Scott et al. [1981], and are interpreted to be late-stage fissure flows. There are a series of small domes on the rift zone, but no elongated vent, fissure, or dome can be unambiguously identified as the source of the flow. Although we only discuss the distal $175 \mathrm{~km}$ of the flow, the total length may be as much as $\sim 500 \mathrm{~km}$. The middle portion of the flow is cut by a $65 \mathrm{~km}$ diameter impact crater at $8.5^{\circ} \mathrm{N}$, $247.3^{\circ} \mathrm{E}$.

[25] We use the 1/64th degree MOLA gridded data provided by the MOLA Science Team. These data have a spatial resolution of $\sim 900 \mathrm{~m} / \mathrm{pixel}$ at this latitude. The relief of this flow over the distal segment we analyze here is significantly greater than the variations in the ambient topography. Thus, even though the flow is north trending, these data are more than adequate to discern the systematic behavior of the flow thickness. Given the north trending orientation of the flow, the extraction of the longitudinal thickness profile from individual MOLA transects by the method of Glaze et al. [2003] is not warranted.

[26] We have compiled 22 topographic profiles perpendicular to the direction of flow (Figure 3 ) across the distal $175 \mathrm{~km}$ portion of the flow. To ensure that these profiles were indeed perpendicular to the flow direction, we generated a local contour map and took profiles that started and ended at a constant elevation. The resultant profiles typically comprise $\sim 20$ pixels across the flow, and show that the flow has an average width of $\sim 15-20 \mathrm{~km}$ and an average thickness of $\sim 50 \mathrm{~m}$. This thickness was determined relative to the best estimate of the ambient topography for each profile.

[27] We also derived a single down-flow profile (Figure 4) which extended down the entire length of the measured portion of the flow. This down-flow profile is used to calculate local gradients for the flow. There is a difference between the slope measured along the center of the flow and that which might be obtained along the edges. However, the absolute values of the slopes are so small, and the extent of the flow is so long, that this factor is inconsequential. For shorter flows with relatively rapid thickening [see, e.g., Mouginis-Mark and Tatsumura-Yoshioka, 1998; Glaze et al., 2003], this consideration could be important.

[28] At certain locations along the flow, it is possible to identify a lava channel running down the approximate center of the flow. This channel is best seen in Figure 5. Our interest here is in the overall volumes of lava contained in the active and passive parts of the flow. At some points along the flow, no channel is evident and thus the entire width of the flow is assumed to be active.

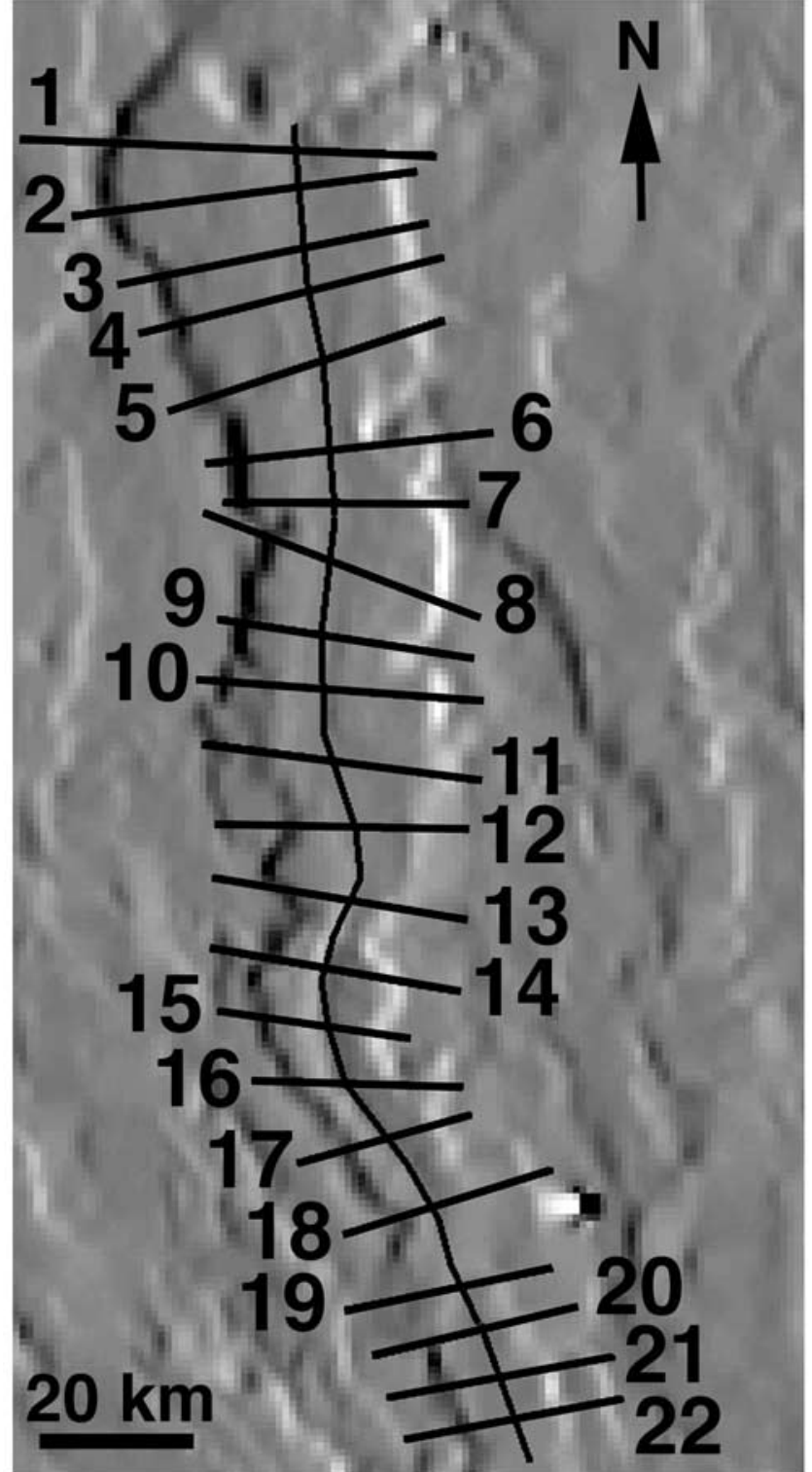

Figure 3. Location of each profile measured in this analysis, using the 1/64th degree MOLA DEM. Numbers refer to individual profiles listed in Table 2. Locations of each profile along the length of the flow are shown as diamonds in Figure 4. Base image is a shaded relief version of the DEM.

Estimates of the volume fraction contained in stagnant margins are based on individual profiles as well as image and gridded topography data for the entire $175 \mathrm{~km}$ extent. Figure 6 shows several typical profiles along the channelized portion of the flow. These profiles indicate that the channel can be as deep as $\sim 25 \mathrm{~m}$ and $5-8 \mathrm{~km}$ wide.

[29] The dimensional data used for the computations appear in Table 2. Each of the transects was inspected to determine the width of the flow at these points and the representative thickness of the flow relative to the ambient topography. Figure 7 graphically illustrates the width and thickness as functions of distance along the flow. The slope 


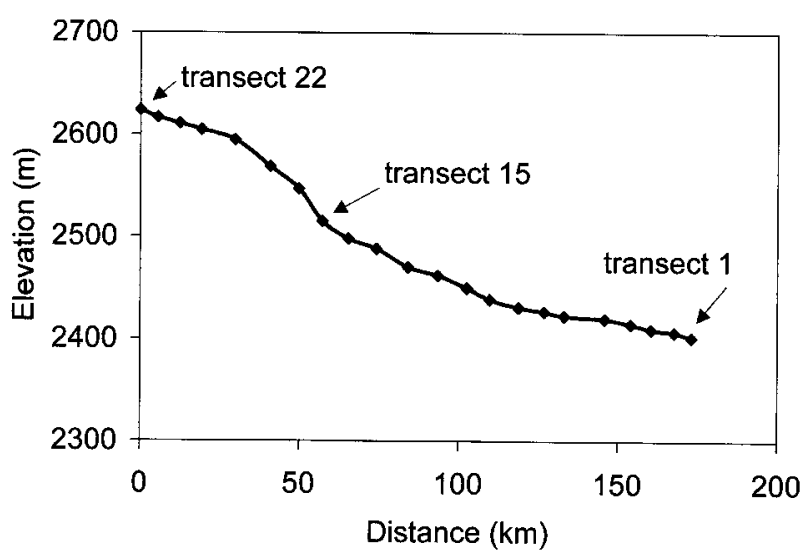

Figure 4. Down-flow profile of the lava flow. Diamonds indicate the location where each perpendicular profile crosses this profile. See Figure 3 for position of this profile with respect to the edges of the flow.

of the underlying topography was estimated from the data presented in Figure 4.

[30] It should be noted that not all transverse profiles are as readily interpretable as those shown in Figure 6. Prudent judgments were made by inspecting each profile to compile the entries into Table 2.

[31] We now turn our attention to developing inferences about the nature of emplacement of the Pavonis flow and the rheologic changes that may have occurred during emplacement. The results of the computations using the dimensional data and the formulas above are shown in Figure 8.

[32] We first consider the case of no losses from the active component, i.e., $L_{m}=\infty$ (lower curve in Figure 8). Thus we use the total width of the flow as the active width. From inspection of Figure 8, we find the unexpected result that there is hardly any increase in the viscosity. There is in fact less than an order of magnitude increase over a distance of almost $200 \mathrm{~km}$. By comparison with large terrestrial basalts, e.g., Mauna Loa 1984, with increases of several orders of magnitude or more over distances an order of magnitude less [Moore, 1987; Crisp et al., 1994; Crisp and Baloga, 1994], such a result was not anticipated.

[33] If this simple model is correct, it indicates that the way the thickening and width increase with distance is essentially what we would normally expect for a coherent viscous flow down a slightly inclined plane with minimal topographic changes along the flow path.

[34] This scenario suggests that flow occurred as a huge, thick, single coherent flow unit. It retained its integrity as it traveled the last $175 \mathrm{~km}$. During emplacement, the flow must have shielded the inner core from significant heat losses that would engender viscosity change due to thermal losses. If this model is valid, then it precludes the emplacement of multiple layers and components, in contrast to the model proposed by Mouginis-Mark and Tatsumura-Yoshioka [1998] for certain long lava flows within Elysium Planitia.

[35] One might be dubious of such a simplistic scenario because there is clear evidence of channelization and thus losses to stationary components during emplacement. Figure 9 shows the relative volume fraction, $V_{m} / V_{d}$ versus $L_{m}$. This curve was computed according to (12) and (13) with the dimensional data in Table 2.

[36] With such a curve, we can pick off the value of $L_{m}$ provided we can estimate $V_{d}$ and $V_{m}$ from the topographic or image data available for a flow. Once we have obtained the value of $L_{m}$, we can use it in (6) to recompute the viscosity change.

[37] It is difficult, of course, to be precise about the volume associated with the stationary components from

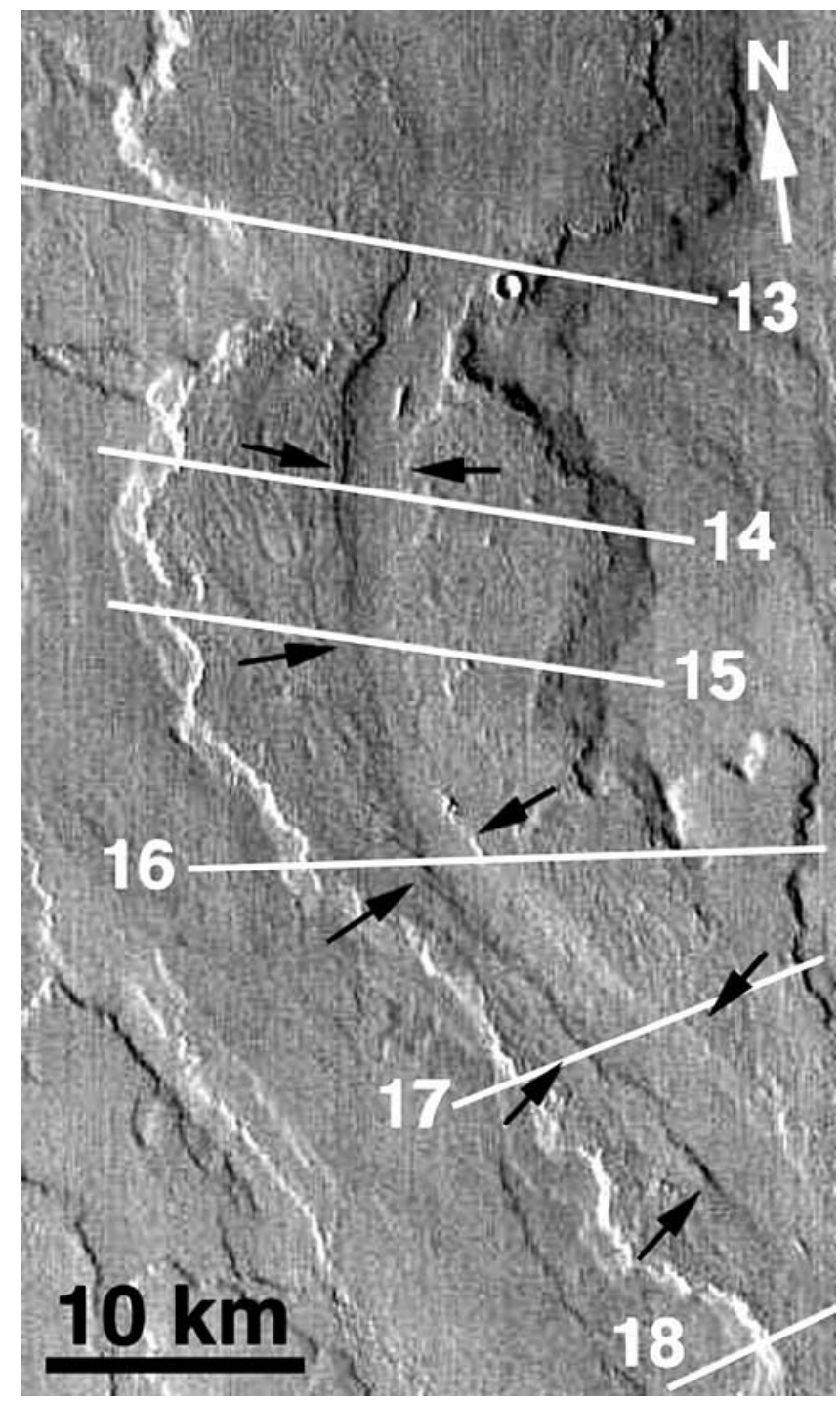

Figure 5. Daytime infrared image, obtained by the Mars Odyssey Thermal Emission Imaging System (THEMIS) instrument, illustrating the channelized part of the flow. The direction of flow emplacement is toward the top of the image (South to North). Black arrows point to the edges of the lava channel, and show that channelization took place along at least $45 \mathrm{~km}$ of the flow length. White lines and numbers correspond to the individual profiles shown in Figure 3. This is part of THEMIS frame number I01739006, which has a spatial resolution of $101 \mathrm{~m} /$ pixel. The local incidence angle is $51.864^{\circ}$. 

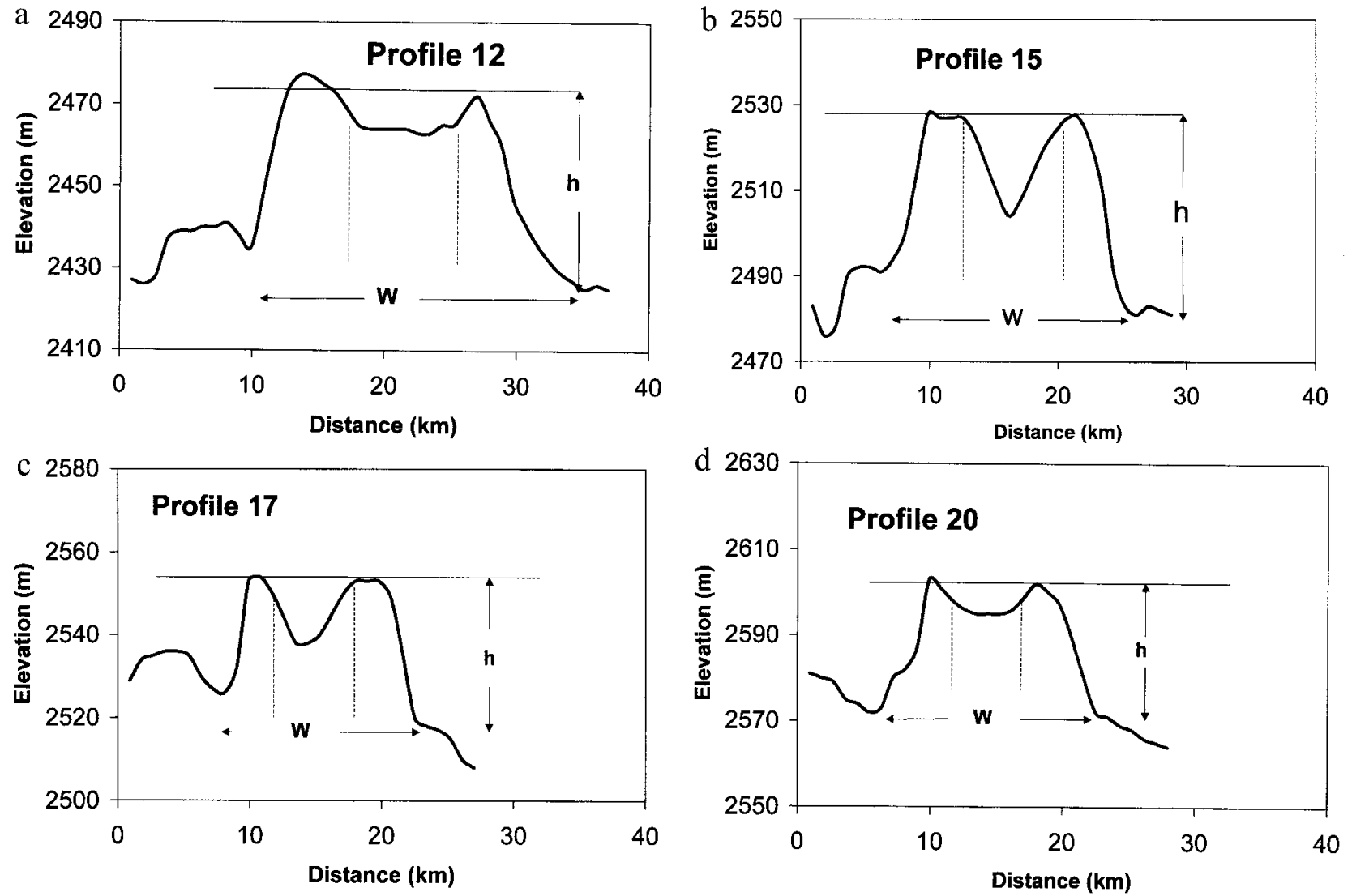

Figure 6. Examples of profiles taken across the channelized portion of the lava flow. Profiles (a) 12, (b) 15, (c) 17, and (d) 20 are shown (see Figures 3, 4, and 5 for location). All profiles are shown at the same scale (40 km along the $x$ axis, and $80 \mathrm{~m}$ along the $y$ axis). Dashed lines indicate plausible estimates of the lava channel/levee boundaries.

image data. We have estimated this fraction in two ways. First we have directly examined the transects from the shaded relief gridded topography data to identify the ratio of the final channel width to the total flow width. We

Table 2. Data on the Lava Flow Northwest of Pavonis Mons

\begin{tabular}{cccccc}
\hline & $\begin{array}{c}\text { Elevation, } \\
\text { Transect }\end{array}$ & $\begin{array}{c}\text { Distance, } \\
\mathrm{km}\end{array}$ & $\begin{array}{c}\text { Slope, } \\
\mathrm{deg}\end{array}$ & $\begin{array}{c}\text { Thickness, } \\
\mathrm{m}\end{array}$ & $\begin{array}{c}\text { Total } \\
\text { Width, km }\end{array}$ \\
\hline 22 & 2624 & 0 & 0.07 & 31 & 17 \\
21 & 2617 & 5.5 & 0.07 & 36 & 17 \\
20 & 2611 & 12.3 & 0.05 & 31 & 17 \\
19 & 2605 & 19.2 & 0.05 & 32 & 19 \\
18 & 2595 & 29.7 & 0.05 & 47 & 19 \\
17 & 2569 & 40.7 & 0.14 & 35 & 14 \\
16 & 2547 & 49.7 & 0.14 & 35 & 23 \\
15 & 2515 & 57.1 & 0.25 & 46 & 20 \\
14 & 2498 & 65.3 & 0.12 & 45 & 19 \\
13 & 2488 & 74.1 & 0.06 & 48 & 18 \\
12 & 2470 & 84 & 0.1 & 48 & 32 \\
11 & 2462 & 93.4 & 0.05 & 49 & 25 \\
10 & 2450 & 102.5 & 0.08 & 62 & 30 \\
9 & 2439 & 109.7 & 0.09 & 53 & 24 \\
8 & 2431 & 118.7 & 0.05 & 61 & 26 \\
7 & 2427 & 126.8 & 0.03 & 62 & 29 \\
6 & 2423 & 133.1 & 0.04 & 60 & 28 \\
5 & 2420 & 145.9 & 0.01 & 57 & 28 \\
4 & 2415 & 154 & 0.04 & 46 & 36 \\
3 & 2410 & 160.4 & 0.05 & 48 & 35 \\
2 & 2407 & 167.6 & 0.02 & 50 & 40 \\
1 & 2402 & 173 & 0.05 & 49 & 40 \\
\hline
\end{tabular}

have used wedge shaped levees as a general formula for estimating the passive volume, although many different shapes are evident in the actual transects. Our estimate by this method gives $\sim 35 \%$ for the volume of lava in stationary components. We have also used a more conservative value corresponding to $\sim 70 \%$ of the volume constructing margins and stagnant zones while the flow was active. From Figure 9, we estimate that the length

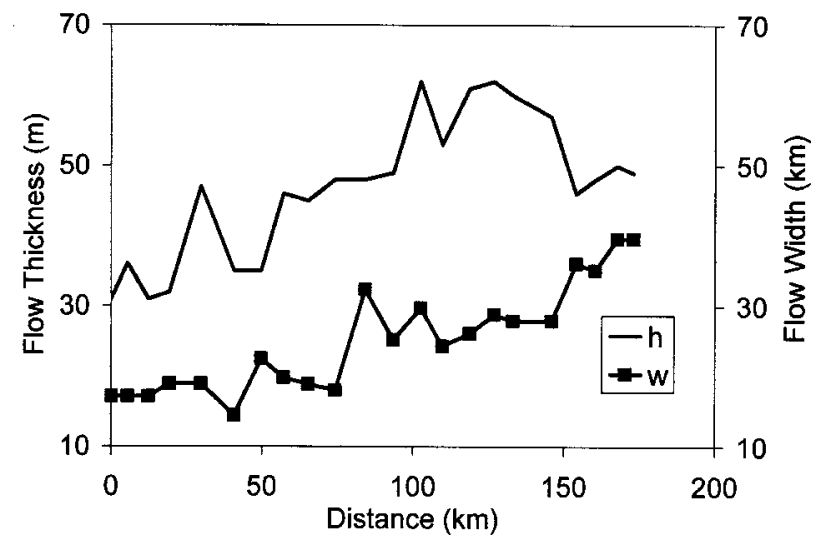

Figure 7. Width and thickness estimates as derived from the 22 cross sectional profiles. 


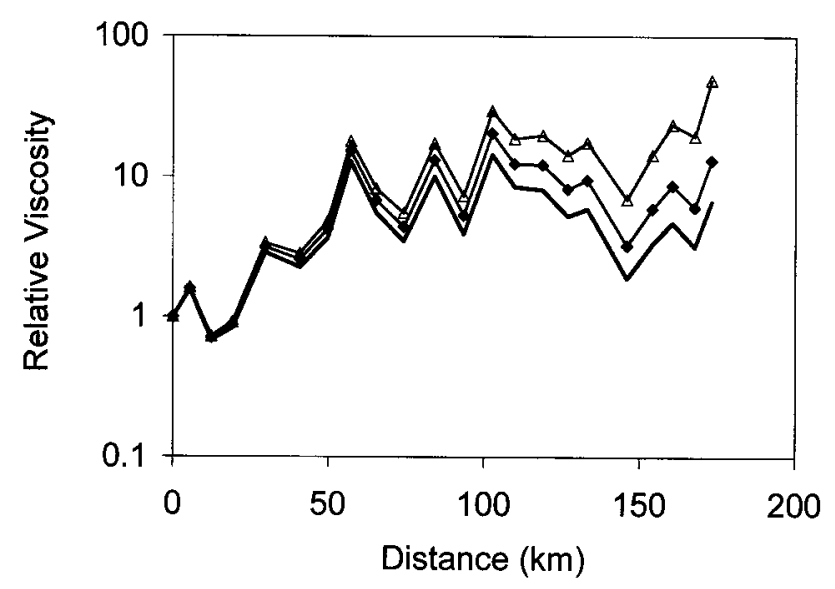

Figure 8. Relative viscosity of the Pavonis flow. Lower curve is for no volume losses. Middle curve (black diamonds) is for $L_{m}=350 \mathrm{~km}$ and uppermost curve (triangles) is for $L_{m}=200 \mathrm{~km}$.

scales corresponding to these two volume fractions are $350 \mathrm{~km}$ and $200 \mathrm{~km}$ respectively. We consider the latter estimate to be extremely conservative on the basis of our estimates of the stationary volume.

[38] With these values of $L_{m}$, we can return to (6) and recompute new viscosity changes. The results are shown as the upper two curves in Figure 8. Except for the most distant segment, the increase in viscosity is only about an order of magnitude over the entire length of the flow.

[39] Even with the volume loss process included, estimates of rheological changes vary little from the case of no margin building. When margin building is taken into account, the dominant variable remains the amount of thickening with distance. And, like many other flows on Mars, there is simply not a great deal of thickening with distance. For the most part, the flow accommodated the shallowing of the underlying slope by lateral expansion and only a very modest thickening. Thus our conclusion is that, although lava was being lost from the active advancing component to the stationary component, this process did not interfere with the dominant dynamics of simple viscous flow.

\section{Discussion}

[40] The analysis above supports the notion that flow advance occurred as a single, essentially coherent lobe. Complex emplacement involving layers and multiple events is not the most likely scenario. Second, none of the physical processes that cause large viscosity increases in terrestrial flows [Moore, 1987; Crisp et al., 1994; Crisp and Baloga, 1994; Baloga et al., 2001] seems to have occurred with the Pavonis flow. We might have expected a larger increase due to radiation from exposed inner core, temperature losses, crystallization, disruption of streamlines and various forms of surface renewal. This does not appear to be the case because the flow does not thicken appreciably.

[41] What must have happened to retain such a relatively constant viscosity therefore is that crustal growth and shielding must have occurred at a rate just about equal to the shedding of lava into the stationary margins. This suggests that a balance existed between the shielding and shedding, which evidently must have been very robust in long lava flows because it seems to occur often on Mars. The ability to maintain such a balance probably depends on low, relatively smooth slopes to avoid vigorous disruption of internal flow streamlines and processes that continually expose the hotter inner core [Crisp et al., 1994; Crisp and Baloga, 1994].

[42] In contrast, many of the flows near Elysium Mons are shorter than the Pavonis flow by a factor of 2-5 and show as much or more of a viscosity increase [Glaze et al., 2003]. This argues for more vigorous discharge, terrain that is rougher on the scale of the flows, or similar factors that could cause higher thermal losses.

[43] If flow advance is governed essentially by simple viscous dynamics, then we are justified in estimating a range of emplacement times for the Pavonis flow. Moore [1987] gives a range of values for the 1984 Mauna Loa flows from $10^{2} \mathrm{~Pa}-\mathrm{s}$ near the vents to $10^{7} \mathrm{~Pa}-\mathrm{s}$ at the front of the $1 \mathrm{~A}$ flow that has been attributed primarily to crystallization, degassing, and the incorporation of solid fragments [Crisp et al., 1994; Crisp and Baloga, 1994]. On the basis of this experience, we consider $10^{5} \mathrm{~Pa}-\mathrm{s}$ to be a typical dynamic viscosity for a thick basalt flow. Using the following values for the Pavonis flow: $\rho=2600 \mathrm{~kg} \mathrm{~m}^{-3}, \theta=0.05^{\circ}$, $w=30 \mathrm{~km}, h=50 \mathrm{~m}, L=173 \mathrm{~km}$, we find a Reynolds number of approximately 5 , a volumetric flow rate of $6 \times$ $10^{6} \mathrm{~m}^{3} \mathrm{~s}^{-1}$, and an emplacement time of only a half a day. At the other extreme, if we consider a dynamic viscosity of $10^{8} \mathrm{~Pa}$-s, then the emplacement time is approximate 500 days and the volumetric flow rate drops by 3 orders of magnitude. Given the large width of the Pavonis flow, the large flow rate of the former case (the lower viscosity) is not unexpected. In the latter case (the higher viscosity), the volumetric flow rate is a reasonable extrapolation of values estimated for some terrestrial eruptions. At present, we know of no way to preclude either of these limits for emplacement times.

[44] Terrestrial experience suggests that the density of lava in large Hawaiian basalt flows can change over the course of an eruption as well as with distance from the vent [Moore, 1982, 1987; Lipman and Banks, 1987]. These and other studies [Einarsson et al., 1949; Cashman et al., 1994]

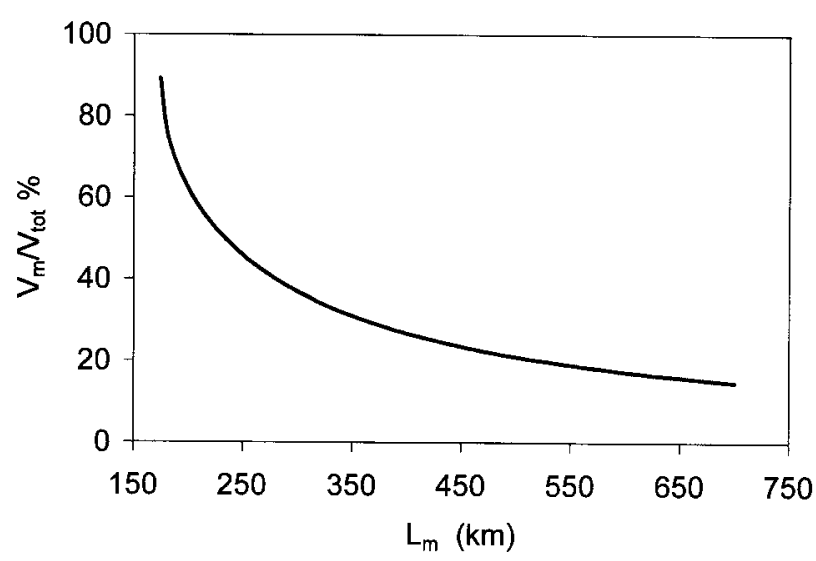

Figure 9. Relative volume of the passive component/total volume versus the range of $L_{m}$ values. 
suggest that differences between erupted and emplaced volumes of lava are quite common in terrestrial basaltic and basaltic andesite flows.

[45] It is therefore tempting to extend our formulation to include an independent loss of volatiles from the active component. This would permit the relative influences of viscosity changes, losses to passive components, and density changes to be resolved. In this scenario volume conservation would be described by

$$
w \frac{\partial h}{\partial t}+\frac{\partial Q}{\partial x}=-\left(\lambda_{m}+\lambda_{g}\right) w_{o} h_{o}
$$

and an independent equation for the conservation of mass might be taken as

$$
w \frac{\partial(\rho h)}{\partial t}+\frac{\partial(\rho Q)}{\partial x}=-\left(\lambda_{m}+\lambda_{g}\right) w_{o} h_{o} \rho
$$

It is interesting that, if the assumption of steady state is now evoked, (14) and (15) require that

$$
\frac{d \rho}{d x}=0
$$

Systematic density changes along the path of the flow, as described by Moore [1982, 1987], Lipman and Banks [1987], and Baloga et al. [2001], thus cannot be built into the approach we have used here without relaxing the simplifying assumptions we have used. The effusion rate of the 1984 Mauna Loa eruption remained essentially constant while time changes in the density occurred [Lipman and Banks, 1987]. Consequently, there are two obvious avenues for extending the approach presented here. First, a quasisteady state that incorporates only time dependence in the density at the vent may provide a more realistic model. Second, permitting a variable rate loss to the stationary margins is more in accord with terrestrial experience.

[46] We can still, however, assess the influence of a simultaneous density change on estimates of viscosity. Corresponding to equations (2)-(4) and (6), we can write

$$
\begin{gathered}
\frac{d Q}{d x}=-\left(\lambda_{m}+\lambda_{g}\right) w_{o} h_{o} \\
Q(x)=Q_{o}\left[1-x / L_{m}-x / L_{g}\right] \\
L_{g}=\frac{Q_{o}}{h_{o} w_{o} \lambda_{g}}=\frac{u_{o}}{\lambda_{g}}
\end{gathered}
$$

and the viscosity formula:

$$
\frac{\nu(x)}{\nu_{o}}=\frac{\sin \theta(x) h(x)^{3} w(x)}{\sin \theta_{o} h_{o}^{3} w_{o}\left[1-x / L_{m}-x / L_{g}\right]} .
$$

At present we do not know how to estimate the parameter $L_{g}$ (or $\lambda_{g}$ ) when stationary margins form and there is a concurrent density change. So we will take the results of our analysis of the Pavonis flow from Section 3 as givens and

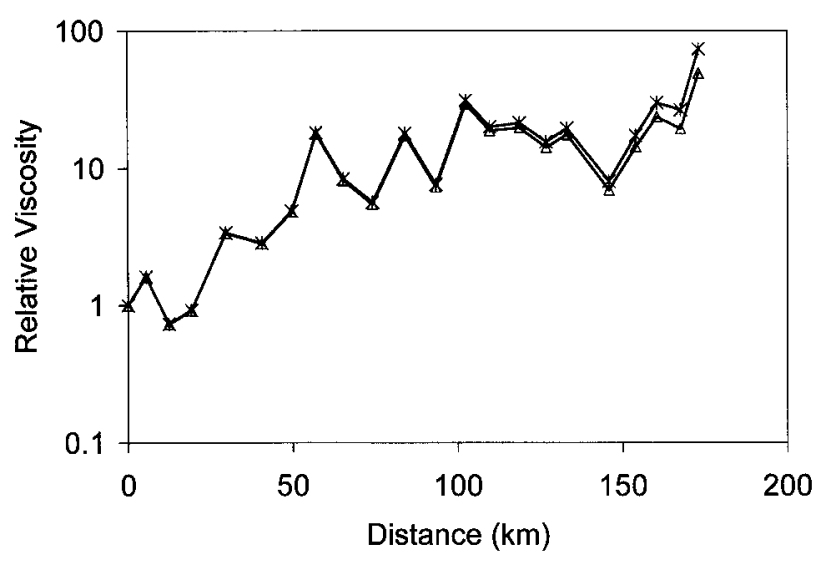

Figure 10. Relative viscosity for Pavonis flow with volumetric flow rate increased by $100 \%$ (asterisks) to account for degassing during flow advance. Both curves assume $L_{m}=200 \mathrm{~km}$. Lower curve (triangles) is the same as in Figure 8.

compare the potential influence of degassing to the constant density case.

[47] When all other things are considered equal, the loss of volatiles during flow means a higher volumetric flow rate is needed to produce the same flow length. To compare the case of degassing to the constant density case, we note that the initial flow rates must satisfy

$$
Q_{d_{o}}\left[1-L / L_{m}-L / L_{g}\right]=Q_{o}\left[1-L / L_{m}\right]
$$

to produce flows of length $L$, where $Q_{d o}$ is the initial flow rate for the degassing case. Thus we can solve for the degassing length scale in terms of the flow rate ratio required to produce the same flow length:

$$
L_{g}=\frac{L}{\left(1-L / L_{m}\right)\left(1-Q_{o} / Q_{d_{o}}\right)} .
$$

Viscosity estimates for the Pavonis flow are shown in Figure 10 for the case when the initial degassing flow rate is twice that for the case with no degassing. Because degassing diminishes the flow rate, the changes in viscosity are systematically higher than the constant density case, as would be expected. However, the difference between the degassing and no degassing cases is small compared to the overall relative change. Again, this indicates that degassing is not important and that this flow must move as a coherent viscous fluid.

[48] Our ability to infer rheologic changes along the flow path still depends critically on our ability to measure or estimate flow thickness as a function of distance. The MOLA gridded topography is adequate for the present task because the flow is a few tens of kilometers in width. When considering the dynamic interaction between the active and passive components, we must be able to discern the relative volumes. Further field study may be helpful in identifying appropriate diagnostics of the different components of flow. There may be textural or other signatures in footprint-scale roughness data from MOLA or in the spectral analysis of 
daytime IR THEMIS data. Nighttime thermal data from THEMIS may also show differences in rock abundances across the flow that can be interpreted in terms of flow structure in areas where dust cover is not a problem.

[49] Perhaps the most stringent assumption we have made in the theory concerns the rate of exchange between the active and stationary components. Here we have used a constant value. But, as noted elsewhere [e.g., Baloga et al., 2001], this is a crude approximation to the behavior of real flows. Efforts are underway to consider the influence of a spatially and temporally varying $\lambda_{m}$ on estimates of rheologic parameters. In addition, it is likely that the material transferred often has different rheologic properties (e.g., material shed from the cooler crust) or a different deformation state (e.g., lava in a state of stress below a yield strength limit). Results of ongoing investigations of these possibilities will appear elsewhere.

\section{Conclusions}

[50] We have presented a method for inferring rheologic changes along the path of a lava flow when there are volume losses from the active component. When dimensional data on channels, levees, and stagnant zones are available, the apportioning of lava between active and passive components can be used to estimate the length scale or rate constant of this process.

[51] Our investigation of the large, approximately $50 \mathrm{~m}$ thick lava flow at Pavonis Mons, indicates only very modest changes in viscosity over the distal $175 \mathrm{~km}$ of flow extent. This conclusion holds whether the processes of margin formation and degassing are included in the analysis or not. The observed thickening and widening of the flow is essentially what would be expected for a single coherent, isothermal, flow on a slightly inclined plane with minor topographic changes along the flow path.

[52] Processes that could have caused major changes in viscosity include direct thermal losses from exposed inner core, overturning and mixing of streamlines due to irregular topography, crystallization and similar phenomena. Because there is evidence of channelization in this flow, such processes must have occurred to some extent. However, they must have occurred with an equilibrium between the formation of crust and cooler outer skin and the shedding of these layers to the stationary margins. This contrasts with the more disrupted emplacement process for thinner Mars flows over more irregular topography that show a significant viscosity increase over much shorter distances [MouginisMark and Tatsumura-Yoshioka, 1998; Glaze et al., 2003].

[53] Large, thick lobate flows that show only modest thickening with distance have been seen in volcanic provinces on Mars since the Viking Orbiter missions [Zimbelman, 1998, and references therein]. Such long lobate 'sheet flows' have been difficult to comprehend as flowing as a single unit with thicknesses of many tens of meters or more. The concept of such extremely thick flows moving as a single coherent units over several hundreds of kilometers has been even more troublesome in the context of relatively low basaltic viscosities. But, such a regime is indicated by our study of the Pavonis flow. This regime features a dynamic balance between the formation of a cooler outer skin and shedding of lava into stationary margins, levees, and stagnant zones. Requirements for attaining such a regime probably include a very thick flow, slopes of only a few tenths of a degree over extended distances, and probably a preexisting surface roughness that is small compared to flow thickness to avoid internal streamline disruption and core exposure during emplacement.

[54] Acknowledgments. The authors would like to thank Drs. James Zimbelman and Scott Rowland for meticulous and thoughtful reviews and $\mathrm{K}$. Shockey for assistance with data reduction. This work was funded by the NASA Planetary Geology and Geophysics (NAG5-7251) and Mars Data Analysis (NAG5-11170) Programs. This is HIGP paper 1285 and SOEST publication 6166 .

\section{References}

Baloga, S., Lava flows as kinematic waves, J. Geophys. Res., 92, 92719279, 1987.

Baloga, S., and D. Pieri, Time-dependent profiles of lava flows, J. Geophys. Res., 91, 9543-9552, 1986.

Baloga, S., L. S. Glaze, J. A. Crisp, and S. A. Stockman, New statistics for estimating the bulk rheology of active lava flows: Puu Oo examples, J. Geophys. Res., 103, 5133-5142, 1998.

Baloga, S. M., L. S. Glaze, M. N. Peitersen, and J. A. Crisp, Influence of volatile loss on thickness and density profiles of active basaltic flow lobes, J. Geophys. Res., 106(B7), 13,395-13,405, 2001.

Cashman, K. V., M. T. Mangan, and S. Newman, Surface degassing and modifications to vesicle size distributions in active basalt flows, $J$. Volcanol. Geotherm. Res., 61, 45-68, 1994.

Crisp, J., and S. Baloga, Influence of crystallization and entrainment of collar material on the emplacement of basaltic aa lava flows, J. Geophys. Res., 99, 11,819-11,831, 1994.

Crisp, J., K. V. Cashman, J. A. Bonini, S. B. Hougen, and D. C. Pieri, Crystallization history of the 1984 Mauna Loa lava flow, J. Geophys. Res., 99, 7177-7198, 1994.

Einarsson, T., G. Kjartansson, and S. Thorarinsson, The Eruption of Hekla, 1947-1948, H. F. Leiftur, Reykjavik, 1949.

Glaze, L. S., and S. M. Baloga, Dimensions of Pu'u O`o lava flows on Mars, J. Geophys. Res., 103, 13,659-13,666, 1998.

Glaze, L. S., S. M. Baloga, and E .R. Stofan, MOLA constraints on lava flow rheologies, Icarus, in press, 2003.

Keszthelyi, L. P., and S. Self, Some physical requirements for the emplacement of long basaltic lava flows, J. Geophys. Res., 103, 27,447-27,464, 1998.

Lipman, P. W., and N. G. Banks, Aa flow dynamics, Mauna Loa 1984, in Volcanism in Hawaii, edited by R. W. Decker, T. L. Wright, and P. H. Stauffer, U.S. Geol. Surv. Prof. Pap., 1350, 1527-1567, 1987.

Malin, M. C., Lengths of Hawaiian lava flows, Geology, 8, 306-308, 1980. Moore, H. J., A geological evaluation of proposed lava diversion barriers for the NOAA Mauna Loa Observatory, Mauna Loa Volcano, Hawaii, and some recommendations, U.S. Geol. Surv. Open File Rep., 82-314, 17 pp., 1982.

Moore, H. J., Preliminary estimates of the rheological properties of 1984 Mauna Loa lava, in Volcanism in Hawaii, edited by R. W. Decker, T. L. Wright, and P. H. Stauffer, U.S. Geol. Surv. Prof. Pap., 1350, 1569$1588,1987$.

Mouginis-Mark, P. J., and M. Tatsumura-Yoshioka, The long lava flows of Elysium Planitia, Mars, J. Geophys. Res., 103, 19,389-19,400, 1998.

Pinkerton, H., and L. Wilson, Factors controlling the lengths of channel-fed lava flows, Bull. Volcanol., 56, 108-120, 1994.

Scott, D. H., G. G. Schaber, K. C. Horstman, A. L. Dial, and K. L. Tanaka, Map showing lava flows in the southwest part of the Tharsis quadrangle of Mars, U.S. Geol. Surv. Misc. Map Ser., I-1268(MC-9-SW), 1981.

Sparks, R. S. J., and H. Pinkerton, Effect of degassing on rheology of basaltic lava, Nature, 276, 385-386, 1978.

Walker, G. P. L., Lengths of lava flows, Philos. Trans. R. Soc. London, Ser. A, 274, 107-118, 1973.

Zimbelman, J. R., Emplacement of long lava flows on planetary surfaces, J. Geophys. Res., 103, 27,503-27,516, 1998.

S. M. Baloga and L. S. Glaze, Proxemy Research, 20528 Farcroft Lane, Laytonsville, MD 20882, USA. (steve@proxemy.com; lori@proxemy.com) P. J. Mouginis-Mark, Hawaii Institute of Geophysics and Planetology, University of Hawaii, 1680 East-West Road, Post 504, Honolulu, HI 96822, USA. 